\title{
Improving the energy efficiency of power transmissions of a rolling stock on the basis of dynamic anisotropy of frictional contact
}

\author{
Vladimir Shapovalov ${ }^{1, *}$, Petr Shcherbak ${ }^{1}$, Pavel Kharlamov ${ }^{1}$, Tatiana Sajamova ${ }^{1}$ and \\ Denis Ryabysh ${ }^{1}$ \\ ${ }^{1}$ Rostov State Transport University, 344038 Rostov-on-Don, Russia
}

\begin{abstract}
The analysis showed the following tendencies of development and energy efficiency of designs of gearboxes for track machines: provision of a maximum level of efficiency, removal of rupture of a stream of the passed capacity, provision of a high level of reliability, decrease of power input on gearbox work, provision of convenience, simplicity of service and steering, engine braking maintenance, and also maintenance of a relatively low cost. The above-mentioned tasks are performed by including clutch pinion gears with the built in frictional amplifier elements in an automatic gearbox, and also during the effect of dynamic anisotropy of frictional bonds. Besides a torque transfer, the clutch gear carries out a safety clutch function. That means that there is sliding at a high rotational power. Factor of strengthening of the given link is $K_{\text {str }} \rightarrow \infty$ due to occurence of dynamic anisotropy of frictional bonds. Physical and mechanical characteristics of contacting surfaces are formed in the course of dynamic interaction of roughnesses in friction units at modes of dry and boundary friction. Essential-nonlinear friction processes, interconnected with dynamic processes on the contact area, define values of target characteristics of frictional system. They can fluctuate in a very wide range. The friction factor $f_{m p}$ for the same friction units at preservation of constant conditions and friction parameters can vary from zero to infinity that is an essence of dynamic anisotropy of frictional bonds.
\end{abstract}

\section{Description of a friction processes research}

Complex automation of power transmissions of land transport (automobile and railway), and also earth-moving machine systems (bulldozers, scrapers, graders, etc.), is one of the most important and actual tendencies of their development. Questions of maintenance of reliability, energy efficiency, safety of operation, and competitiveness of the above-named systems are inseparably linked with reliability and efficiency of their power transmissions. Now all industrially developed countries are actively creating unmanned energy-efficient technologies of steering in land mobile systems. Intensive researches and practical

\footnotetext{
* Corresponding author: tmt@rgups.ru
} 
workings-out in the given direction are conducted on a railway transportation, too. In particular, authorities of plc. $\mathrm{RZhD}$, have decided on transference of all specialised rolling stock on automatic control.

Technical and economic characteristics of modern land transport and technological means (LTTM) are closely connected with reliability and energy efficiency of their power transmissions. According to tasks in view, they should have the automatic mode of steering providing convenience of operation and maintenance of LTTM, a minimum level of loss of power during movement, and also during transition from drive to drive, maintenance of convenience, simplicity of service and control.

We have found out technical solutions of some practical problems on the basis of fundamental researches in the field of dynamics of machines, contact interaction of solids, and tribology, and also with the help of fundamental theoretical bases of anisotropy of frictional bonds. These solutions allowed creating a design of an automatic gearbox with technical and economic characteristics at the level of the best world samples.

The theoretical basis of these practical solutions has been formed by fundamental researches in the field of anisotropy of frictional bonds, in particular, dynamic anisotropy [1]. The transfer function connecting power interactions of contacting roughnesses in a normal and tangential direction was offered by Coulomb and Amontons in 1699 in the form of the relation of their average values $f_{f r}=\frac{F_{\text {fr.average }}}{N_{\text {average }}}$ (Fig. 1). As the researches of friction processes have shown, the transfer function does not reflect the real values of a friction factor $f_{f r}$, that are defined by dynamics of formations of adhesive and deformation bonds during elastic and dissipative friction processes.

Value of a friction factor varies from 0 to $\infty$; it is dynamics of frictional interaction of friction pairs that defines real values $f_{\text {fr }}$, the level of energy dissipation, and the level of losses on friction, i.e. the efficiency factor of a frictional bond. Representation of a transfer function (a friction factor) of a frictional bond in the form of the relation of spectral functions (1) allows receiving real values of a friction factor, considering ratio of elastic and dissipative processes as well as predicting possibility of occurrence of frictional selfoscillations. It also helps appear the phenomenon of dynamic anisotropy in reactions of frictional bonds.

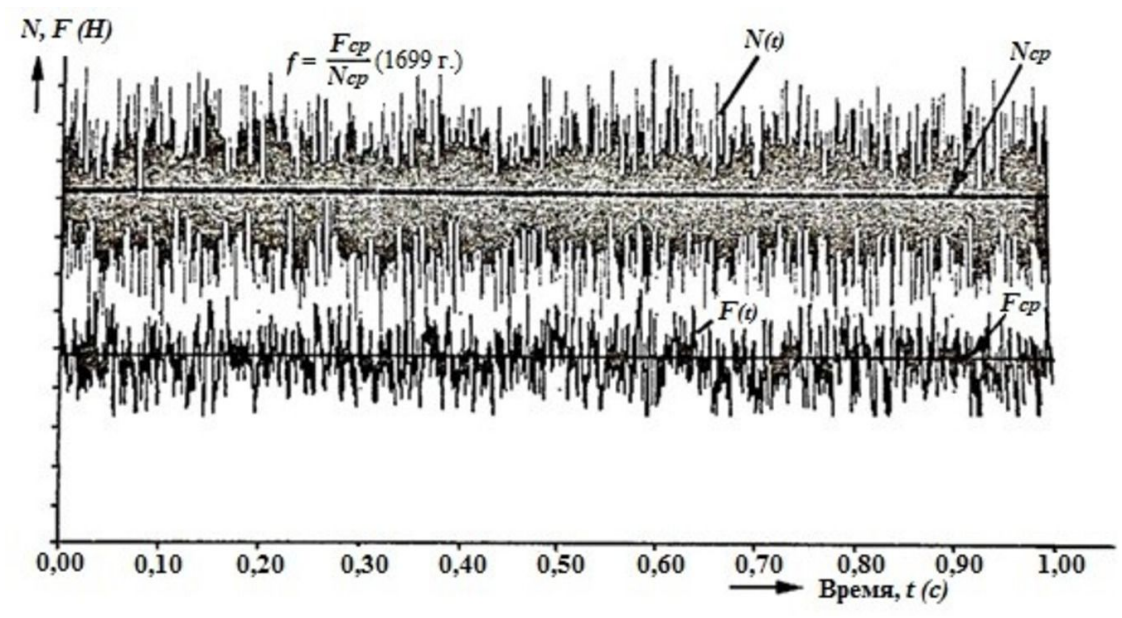

$N(t), F(t)$ - variations of forces of contact interaction in normal and tangential directions concerning a stationary trajectory in time $t ; N_{a v}, F_{a v}$ are average values of forces of contact interaction in normal and tangential directions.

Fig. 1. Dependence between average values of normal force and friction force: 


$$
f_{f r}=W_{f r}(P)=\frac{S_{x y}}{S_{y y}}
$$

where $S_{x y}$ is mutual spectral function of tangential and normal force interaction of contacting micro-and macro-roughnesses; $S_{y y}$ is autospectral function of normal force influence on the frictional contact area $[2,8]$.

Process of control by clutch-pinion gears with the built in intensifying links is offered to be performed on the basis of methods of dynamic monitoring of the processes taking place in a frictional system. For example, it is "track-rolling stock" for the track machine. The information on the current value of a sliding speed of a car wheel in relation to a rail is represented in the form of the mutual relation and the relation of autotribospectrums which are recorded by equipment and passed to the system of automatic regulation (SAR) for the analysis [3].

Processes of research, preliminary treatment, energy saving, monitoring and optimisation of frictional mechanical systems, are connected with the solution of complex nonlinear differential equations systems. In its turn, for the purpose of simplification of process of search of optimum solutions, researchers are compelled to make linearization of mathematical models that are essential-nonlinear interconnected processes and the phenomena. Thus, they break a principle of unacceptability of a superposition principle for nonlinear systems.

The solution of the mentioned-above contradiction consists in carrying out of long-term and expensive numerous natural tests or in application of methods of physical and mathematical modelling. This position is defined by the following circumstances: target frictional characteristics are complex and non-linear, they depend on a great number of internal and external factors. The dynamic processes produce essential mutual impact taking place at the frictional contact area in a quazi-linear mechanical subsystem of a frictional mechanical system. Therefore, target characteristics can change 10 times and more, and the transfer function of the frictional contact area or its analogue in the form of a friction factor can change from zero indefinitely.

Let us consider the analogue of the process of clutch of friction plates in case of preliminary pressing of butts in samples 1 and 2 (Fig. 2 a) and at the subsequent elimination of normal force $(N=0)$ (Fig. 2 b). Sample 2 will be kept on sample 1 by a friction force, i.e. $F_{f r}=P=m \cdot g$, hence:

$$
f=\frac{F_{f r}}{N}=\frac{m g}{0} \rightarrow \infty
$$

In case of influence on contacting surfaces of friction by external dynamic strengthening, as a result of a resonance of dynamic processes, i.e. coincidence of bearing frequency (or the basic frequency of tribospectrum) with frequency of external dynamic influence, the value of a friction factor aspires to 0 [4]:

$$
f=\frac{F_{f r}}{N}=\frac{0}{N} \rightarrow 0 .
$$

The given status of frictional contact area is known as Tolstoy - Push effect. It is widely used in various areas of science and technics. Active working bodies of building and track machines, vibrating influence on working sides of the metalcutting equipment, vibrotamping of ballast by track machines. 


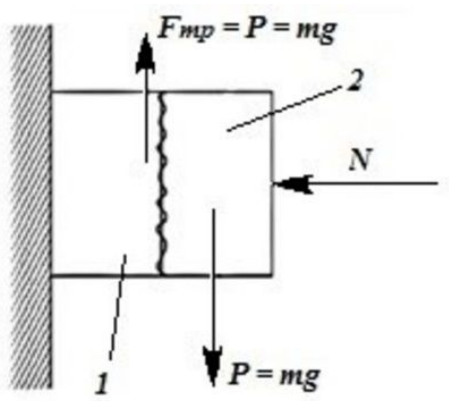

a)

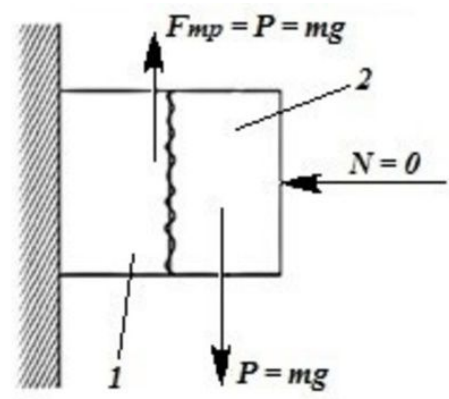

b)

Fig. 2. Contacting friction surfaces

The above-named anisotropic statuses of the frictional contact area are widely known in the engineering (i.e. $f \rightarrow 0 ; f \rightarrow \infty$ ), they arise in uncontrollable unspinning of nuts of threaded connections. During dynamic influence there is self-unspinning of threaded connections (i.e. at $f \rightarrow 0$ ); thus, jamming conditions take place in threaded connections, when friction forces on contacted sides of coils of a thread are sure to exceed climbing resistance. There are known cases of transition in uncontrollable movement of the rolling stock fixed - by brake shoes, jammings and collapses of toothed gearings in a mode of oil lack (i.e. $f \rightarrow \infty$ ) etc.

Thus, we have three statuses of a frictional system:

I) $\mathrm{F}_{\mathrm{fr}}>\mathrm{F}_{\mathrm{sl}}-\mathrm{a}$ jamming status;

II) $\mathrm{F}_{\mathrm{fr}}=\mathrm{F}_{\mathrm{sl}}-\mathrm{a}$ status of unstable balance;

III) $\mathrm{F}_{\mathrm{fr}}<\mathrm{F}_{\mathrm{sl}}-$ a slippage status.

In case of the unstable balance $N \sin \alpha=N \cos \alpha(f \pm \Delta f)$ (Fig. 3), and considering the presence of fluctuations of a friction factor $\Delta f$ in a range from 0 to $\infty$, there is a possibility to steer the frictional system by value change $\Delta f$, transferring the system in a mode of jamming I or in a mode of slippage III.

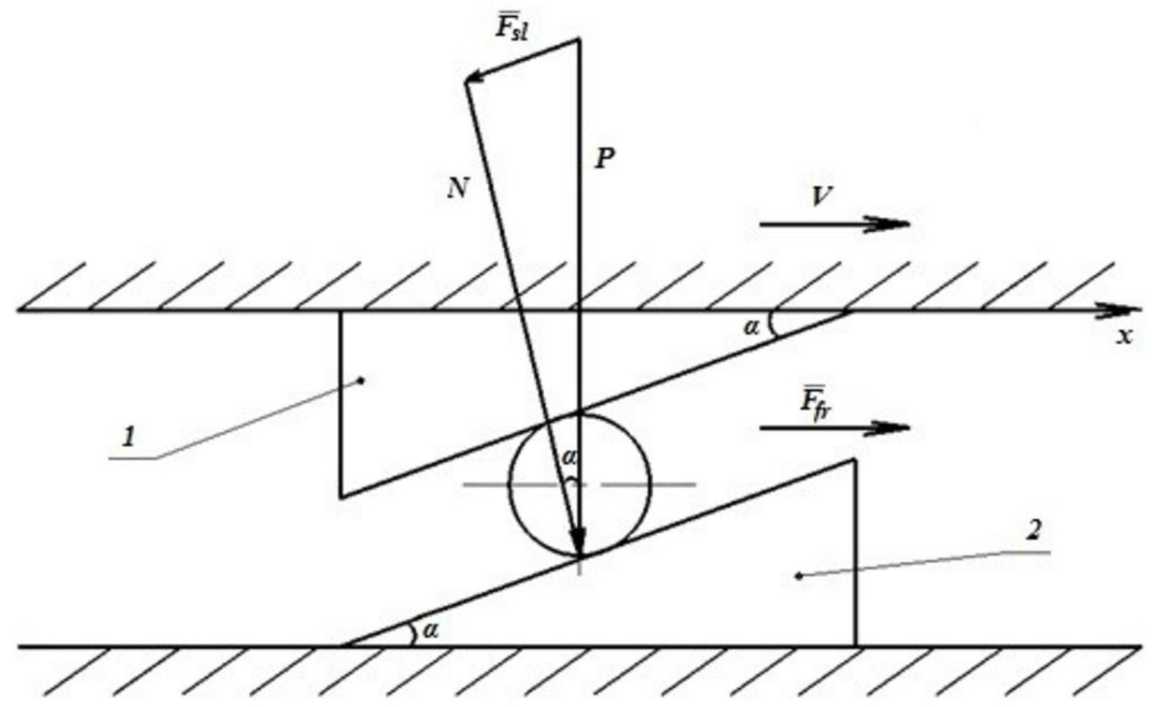

1 - loading path of an anchor, 2 - loading path of a switching-on disk

Fig. 3. The scheme of the mechanism of switching-on of a clutch-pinion gear 


\section{Description of a clutch-pinion gear}

The design of a clutch-pinion gear (Fig.4) represents a pinion gear with the frictional multidisk clutch with an intensifying link in its body. The clutch-pinion gear works as follows. When voltage is fed on an electromagnet, floating disks 6 and an anchor 8 are drawn by forces of the electromagnetic clutch to the leading semiclutch. The switching-on disk creates the effort of inclusion on surfaces of leading and conducted friction plates 4, being turned on loading paths of an intensifying link concerning an anchor:

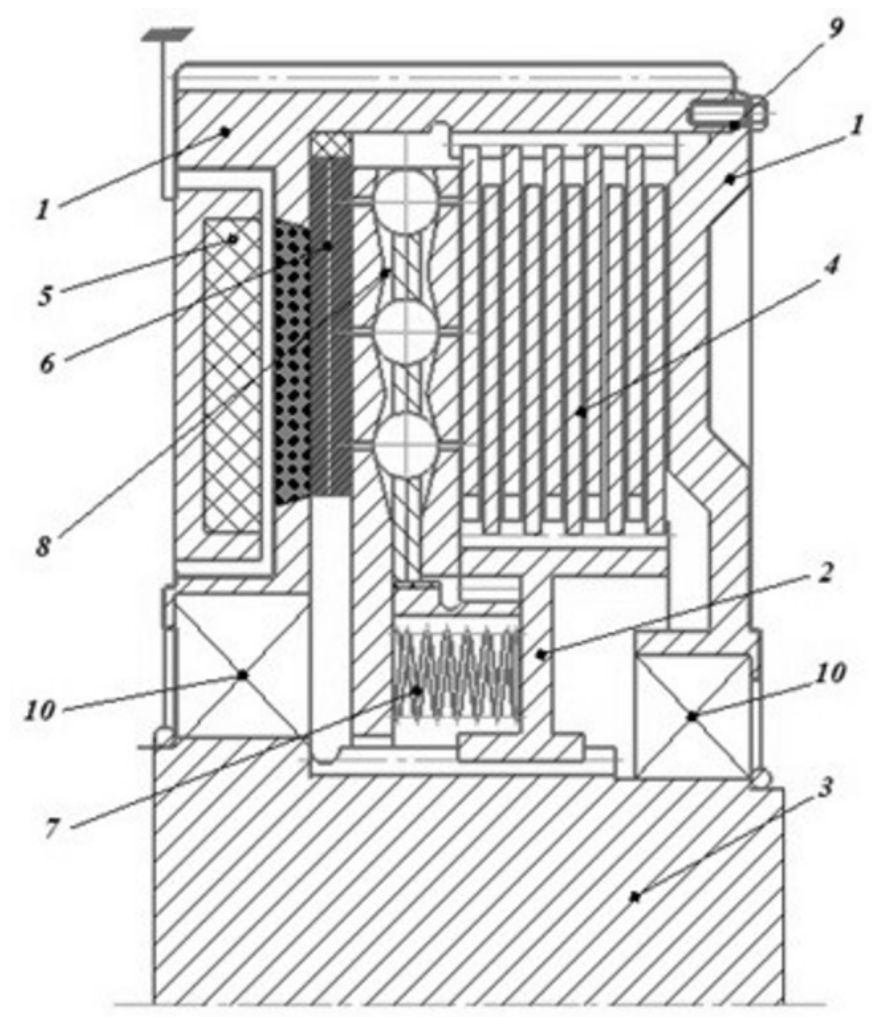

1 - leading semiclutch; 2 - conducted semiclutch; 3 - shaft; 4 - package of friction plates; 5 - electromagnet; 6 - floating disks; 7 - returnable spring; 8 - disk of switching-on and a rolling motion body; 9 - a bolt; 10 - bearing.

Fig. 4. A clutch-pinion gear

The package $n$ of stabilising floating disks 6 is applied in a design of a clutch-pinion gear; it is intended for obtaining demanded value of a friction factor between them, that is $f_{f r}=f_{\max }$ or $f_{f r}=f_{\min }$ at $\Delta f \rightarrow 0$. The number of disks $n$ is defined according to probability of $f_{\min }$ in the formula value $n=\frac{1}{B}$.

To be able to obtain a maximum level of a strengthening factor $K_{s t r} \rightarrow \infty$ in the strengthening link, the discharge angles $\alpha$ of loading paths of surfaces of the anchor, and the disk of switching-on 8 satisfy the condition of the guaranteed inclusion of a clutch, i.e. $F_{f r} \geq F_{s l}$ at $\alpha=f_{f r}+\Delta f$ (see Fig. 3). Then, a condition of $K_{s t r} \rightarrow \infty$ is value maintenance $f_{f r}$ in a package of friction plates 6 at $\Delta f \rightarrow 0$. Taking into consideration the power ratio in terms of presented on Fig. $3 F_{s l}=P \cdot \sin a \cdot \cos a, F_{f r}=P \cdot f_{f r}$, reaching $K_{s t r} \rightarrow \infty$ is possible when $F_{f r}=F_{s l}$ then the friction factor should be equal to $f=\sin a \cdot \cos a$. 
The factor of strengthening of the given link $K_{s t r} \rightarrow \infty$ is possible due to dynamic anisotropy of frictional bonds, i.e. changes of values of a friction factor $f$ for the same contacting materials and parameter $P V$ (where $P$ is loading, $V$ is sliding velocity) from 0 to $\infty$. The minimum value $f \rightarrow 0$ is obtained under condition of power electromagnetic resonant influence on contacting surfaces (Tolstoy - Push effect):

$$
N_{e l}=F_{e l} \cdot \sin \omega_{p} t
$$

where $\mathrm{F}_{\mathrm{el}}$ is force of electromagnetic clutch, $\omega_{p}$ is resonant frequency of frictional contacting,

$$
\omega_{p}=\sqrt{\frac{C_{k}}{m_{\Sigma}}}
$$

where $C_{k}$ is total ruggedness of a frictional contact area, $m_{\Sigma}$ is total active volume of weights of contacting micro-and macroroughnesses.

The frictional pinion gear clutch switches on at voltage feed, $U_{o n}=U \sin \left(\omega_{p} t+180^{\circ}\right)$ where $\omega=\omega_{p}$ is frequency of external antiresonant influence on contacting micro-and macroroughnesses, $\omega_{p}$ is bearing frequency of a tribospectrum in a normal plane. As a result of dynamic influence, conditions $f \rightarrow \infty$ (see Fig. 5) are created.

The equality $f_{\min }=\sin \alpha \cdot \cos \alpha$ creates a condition of appearing of a powerful intensifying link on the basis of frictional servoeffect. Introduction of dynamic resonant $U_{O N}=U \sin \omega_{p} t$ or antiresonant influence $U_{O F F}=U \sin \left(\omega_{p} t+180^{\circ}\right)$ in a control system with a bearing frequency of a tribospectrum in a normal plane $\omega_{p}$, provides the guaranteed inclusion and clutch-pinion gear disconnection. It is a basis of its eliability and energy efficiency, as the minimum level of expenses of capacity $\left(P_{y} \rightarrow 0\right)$ on control of a clutchpinion gear as $K_{s t r}=\frac{P}{P y}$ and at conditions $K_{\mathrm{y}} \rightarrow \infty$ capacity $P_{y} \rightarrow 0$ is provided.
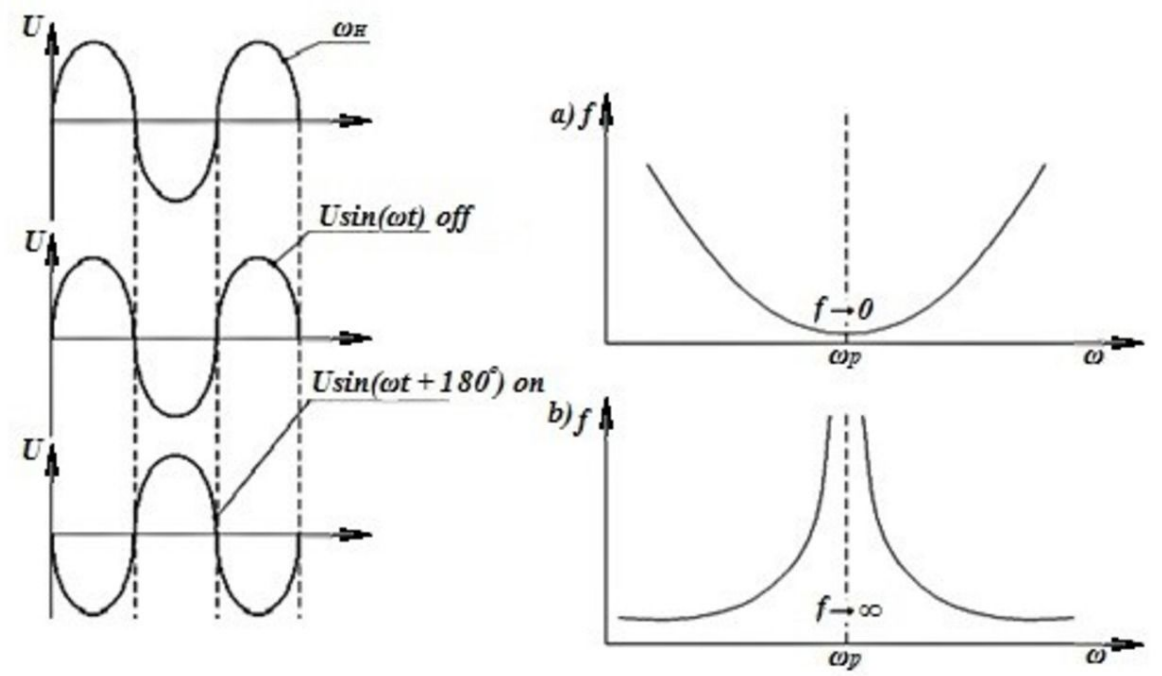

Fig. 5. Schedules of steering dynamic interaction on a frictional contact area.

During switching on of leading and conducted friction plates, the twisting moment on a driven shaft is increasing progressively and is proportional to mutual pressing of friction surfaces those also increase in force. It allows connecting shafts under loading and with a 
considerable initial difference of their angular speeds. In the course of switching-on, the clutch revolves, and main shaft dispersal is carried out smoothly, without blows.

\section{Conclusion}

As an example of use of a clutch-pinion gear, we will examine a variant of scheme of automatic gear case intended for introduction by a track machine MTP6-K (Fig. 6). During automatic control of a step-by-step gearbox, the choice of the moment of inclusion of this or that drive is provided with the accounting a minimum of three information parameters, such as loadings of the engine, speed of movement, and the drive which have been switched on at the moment of switching [5-7]. Besides, in a control system of a gear case there should be provided a possibility of correction of the law of a gear shift depending on speedup developed by the vehicle [9-10].

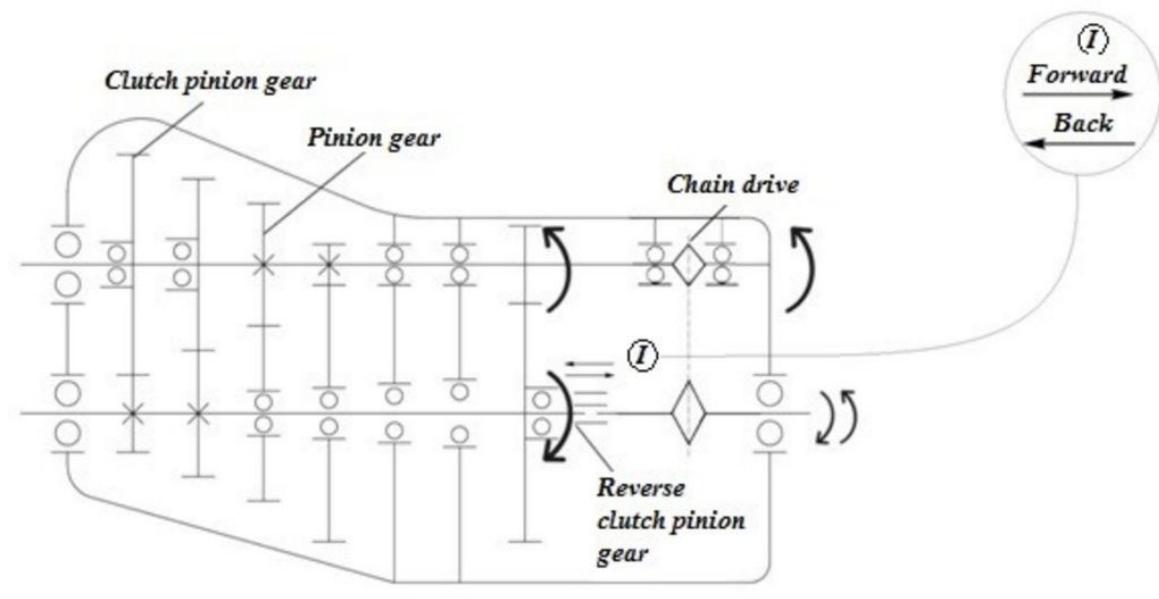

Fig. 6. The scheme of an automatic gear case of a gear shift.

The automatic gear case excludes rupture of a stream of power at transition from the least drive on the increasing one. The change occurs in the beginning of the switching on of the following drive while the previous one has not been disconnected yet. After the following clutch-pinion gear is switched on without rupture of a stream of power, there is a disconnection of the previous clutch-pinion gear. Process of switching of drives, in particular, transition to a higher drive, for example, transition from I to II is carried out by switching of an electromagnet of the second drive with the switched on first drive. At the moment when the anchor is connected with a leading semiclutch by forces of electromagnetic clutch, and it "will catch up" with a disk of switching on, and shafts of loading paths (see Fig. 3), there will be a moment of disconnection of an electromagnet of a clutch-pinion gear of the I drive between them. On Fig. 7 the scheme of transition from igear to the increasing one is presented during a smooth change of a transmission ratio at the expense of relative slippage of disks, thus, rupture of a stream of power is excluded.

For an exception of occurrence of a mode of behaviour of elements of automatic gear case in a mode of the "closed" power circuit and creation of possibility of collapse of 2 pinion gear teeth provided that $i \neq 1$ the scheme of electronic locking of the given mode is provided in a control system. 


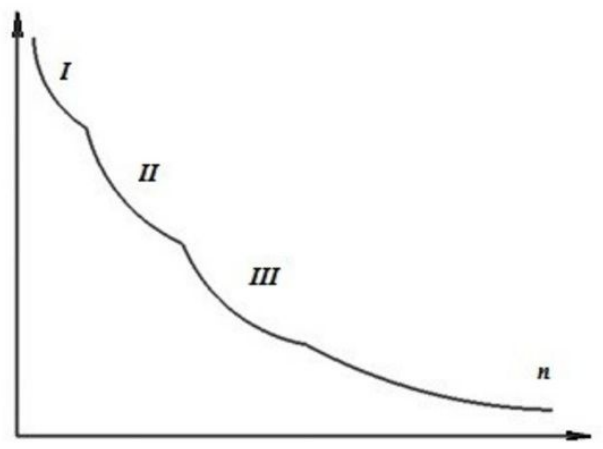

Fig. 7. The scheme of transition from i-drive to the increasing one.

At transition from i-drive to i-1 (reducing gear) there is a power-off of all $n$ electromagnets, including i clutch-pinion gears. In this connection, all clutches are switched-off, power transmission is disconnected from the engine, the moment on the output shaft is equal to 0 . After fixing of the given position by a torque sensor, there is a switching on of an electromagnet of a clutch-pinion gear i-1. Critical parameter of automatic gear case defining its economic efficiency, in particular, specific fuel consumption, is the value of efficiency.

Applying a two-shaft system as a basic scheme of automatic gear case allows using all drives from the first to $n(7 \div 10)$ by using only one gearing with the level of value of efficiency up to 0.92 . This system possesses the maximum level of value of energy efficiency, in comparison with already known technical solutions.

Researches are conducted within realization of a grant of JSC «Russian Railways» on development of scientific and pedagogical schools in the field of railway transport.

\section{References}

1. V.V. Shapovalov, Patent of Russia №2016128401 (2018)

2. V.V. Shapovalov, Patent of Russia №2517946 (2014)

3. V.V. Shapovalov, Patent of Russia №2343450 (2009)

4. S.A. Kharitonov, Automatic gear boxes (Open Company «Publish house Astrelj», Moscow, 2003)

5. J.N. Kudrjashev, Multiline drives of differential type (Mechanical engineering, Moscow, 1981)

6. V.V. Shapovalov, Application of methods of physical and mathematical modelling and tribospectral identification for monitoring of frictional mechanical systems (Mechanical Engineering Bulletin, Moscow, 2009)

7. A.P. Petrov, Modern designs of automatic gear cases (Publishing house of Kurgan State University, Kurgan, 2015)

8. A.A. Loktev, Simulation of the railway under dynamic loading (Contemporary Engineering Sciences, Moscow, 2015)

9. M. Dumitriu, Modeling of railway vehicles for virtual homologation from dynamic behavior perspective (Applied Mechanics and Materials, 2013)

10. Y.C. Yang, Estimation of Heat Flux and Temperature Distributions in a Composite Strip and Homogeneous Foundation (Int. Commun. Heat Mass Tran., 2010) 